\title{
OPEN Bimanual digit training improves right-hand dexterity in older adults by reactivating declined ipsilateral motor-cortical inhibition
}

\author{
Eiichi Naito ${ }^{1,2 \otimes}$, Tomoyo Morita ${ }^{1,2,3}$, Satoshi Hirose ${ }^{1,4}$, Nodoka Kimura ${ }^{1}$, Hideya Okamoto ${ }^{5}$, \\ Chikako Kamimukai ${ }^{5}$ \& Minoru Asada ${ }^{1,3,6}$
}

Improving deteriorated sensorimotor functions in older individuals is a social necessity in a superaging society. Previous studies suggested that the declined interhemispheric sensorimotor inhibition observed in older adults is associated with their deteriorated hand/finger dexterity. Here, we examined whether bimanual digit exercises, which can train the interhemispheric inhibitory system, improve deteriorated hand/finger dexterity in older adults. Forty-eight healthy, right-handed, older adults (65-78 years old) were divided into two groups, i.e., the bimanual (BM) digit training and righthand $(\mathrm{RH})$ training groups, and intensive daily training was performed for 2 months. Before and after the training, we evaluated individual right hand/finger dexterity using a peg task, and the individual state of interhemispheric sensorimotor inhibition by analyzing ipsilateral sensorimotor deactivation via functional magnetic resonance imaging when participants experienced a kinesthetic illusory movement of the right-hand without performing any motor tasks. Before training, the degree of reduction/loss of ipsilateral motor-cortical deactivation was associated with dexterity deterioration. After training, the dexterity improved only in the BM group, and the dexterity improvement was correlated with reduction in ipsilateral motor-cortical activity. The capability of the brain to inhibit ipsilateral motor-cortical activity during a simple right-hand sensory-motor task is tightly related to right-hand dexterity in older adults.

Sensorimotor functions deteriorate in older adults ${ }^{1,2}$. Thus, improving these functions in older adults is a social necessity in any super-aging society. Hand/finger dexterity is a representative sensorimotor function that enables the performance of daily skillful manual behaviors. Among primates, humans have well-developed hand/ finger dexterity, in which the primary motor cortex (M1) plays a particularly important role ${ }^{3-6}$. Similar to many sensorimotor functions, human hand/finger dexterity is also deteriorated by aging ${ }^{7-9}$. Therefore, improving deteriorated hand/finger dexterity in older individuals is a worthwhile challenge.

Several studies have reported that reduced transcallosal interhemispheric inhibition from the contralateral to the ipsilateral $\mathrm{M}^{7}$ and hyperactivation of the ipsilateral primary sensorimotor cortex (SM1) in older adults ${ }^{8}$ are associated with deteriorated dexterity of their right hand, as evaluated by peg task performance. In addition, the aging-related reduction of interhemispheric inhibition exerted from the contralateral (left) M1 may have contributed to the age-related reduction/loss of ipsilateral (right) M1 deactivation during a right-hand sensorymotor task ${ }^{10}$. Thus, these lines of evidence suggest that the reduction/loss of ipsilateral M1 deactivation during a right-hand sensory-motor task, likely caused by the reduction of interhemispheric inhibition from the left to right $\mathrm{M} 1$, deteriorates right hand/finger dexterity in right-handed older individuals.

\footnotetext{
${ }^{1}$ Center for Information and Neural Networks (CiNet), Advanced ICT Research Institute, National Institute of Information and Communications Technology (NICT), 1-4 Yamadaoka, Suita, Osaka 565-0871, Japan. ${ }^{2}$ Graduate School of Frontier Biosciences, Osaka University, 1-3 Yamadaoka, Suita, Osaka 565-0871, Japan. ${ }^{3}$ Institute for Open and Transdisciplinary Research Initiatives, Osaka University, 1-1 Yamadaoka, Suita, Osaka 565-0871, Japan. ${ }^{4}$ Otemon Gakuin University, Faculty of Psychology, 2-1-15, Nishiai, Ibaraki, Osaka, Japan. ${ }^{5}$ Element Technology Research \& Development Section, Global Research \& Development Department, , Mizuno Corporation, 1-12-35 Nanko-kita, Suminoe-ku, Osaka, Osaka 559-8510, Japan. ${ }^{6}$ International Professional University of Technology in Osaka, 3-3-1 Umeda, Kita-ku, Osaka 530-0001, Japan. ${ }^{\varpi}$ email: eiichi.naito@nict.go.jp
} 


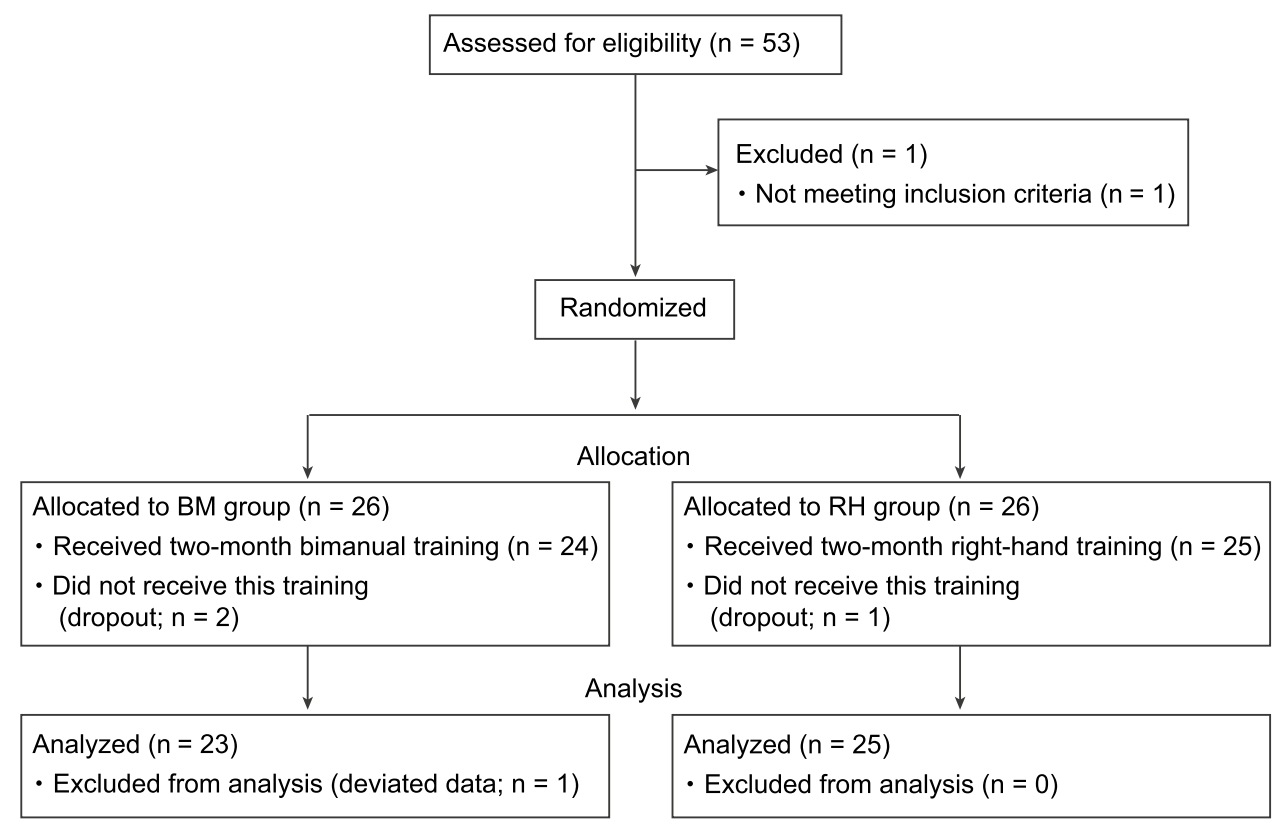

Figure 1. An experimental flow chart in the present study.

In the present study, first, we confirmed the deterioration of right hand/finger dexterity using a peg task and the reduction/loss of ipsilateral deactivation using a right-hand sensory-motor task (independent from the peg task) in 48 healthy, right-handed, older adults (65-78 years old) compared with 31 younger adults (20-27 years old). Kinesthetic illusion was used as a right-hand sensory-motor task. We measured brain activity using functional magnetic resonance imaging (fMRI) while the blindfolded participants experienced kinesthetic illusory flexion of the right stationary hand elicited by a muscle afferent input during its tendon vibration ${ }^{11}$. We know that, during the illusion, the hand/arm sections of the contralateral (left) SM1 and of the dorsal premotor cortex (PMD) are activated by receiving the kinesthetic input passively, while the ipsilateral side is deactivated ${ }^{11}$, presumably via interhemispheric inhibition from the left to right SM1-PMD. One of the advantages of this task is that it allows the evaluation of the pure state of the interhemispheric inhibitory system by measuring ipsilateral SM1-PMD deactivation (negative blood oxygenation level-dependent [BOLD] signal) in individual participants, with no motor tasks being performed.

Next, prompted by previous reports ${ }^{7,8}$, we examined whether the reduction/loss of ipsilateral M1 deactivation observed during the illusion is associated with dexterity deterioration in the older participants. Here, we formulated a specific anatomical hypothesis for the M1 region. Our recent developmental fMRI study showed that deactivation in a particular region of the hand/arm section of the ipsilateral (right) M1 (peak coordinates $\mathrm{x}, \mathrm{y}, \mathrm{z}=36,-26,66)$ during a simple right-hand sensory-motor task is better developed in children with higher right hand/finger dexterity ${ }^{6}$. In the present study, we hypothesized that this relationship between deactivation and dexterity observed during childhood is also present in older adults in this particular region. To test this hypothesis, we set a region-of-interest (ROI) in this M1 region (M1 ROI; 4-mm radius sphere around the M1 peak).

Given that the reduction/loss of ipsilateral M1 deactivation (inhibition) is one of the causes of the deterioration of hand/finger dexterity in older adults, their hand/finger dexterity might be improved by reactivating the ipsilateral inhibition, i.e., via interhemispheric inhibition from the left to right M1. Therefore, intensive daily training was carried out by the older participants for approximately 2 months (see Supplementary Methods and Tables S1-3), during which we checked the progress of their training on a weekly basis. The older participants were divided into the following two groups (Fig. 1). One group performed bimanual digit training (BM group; $\mathrm{n}=23$; see Table S1). Bimanual training was chosen because it likely facilitates transcallosal neuronal communication between the two M1 regions ${ }^{12,13}$. During this training, the participants performed both the same and different finger actions using the two hands. In the case of different actions, each of the left or right M1 had to control disparate actions simultaneously while mutually inhibiting the potential occurrence of synchronized actions $^{14}$, probably through the transcallosal interhemispheric inhibitory system. Another group underwent unimanual digit training using the right-hand ( $\mathrm{RH}$ group; $\mathrm{n}=25$; see Table $\mathrm{S} 1$ ). This group performed the same exercises performed by the BM group but used the right-hand exclusively. Thus, this group intensively trained the right-hand only, and we expected that the interhemispheric inhibition could be better trained in the BM group. Importantly, neither group was trained in the peg task per se during the training period, to avoid pegtask-specific training effects.

After the training, using the peg task, we examined whether the right hand/finger dexterity was improved in the BM group, and whether such behavioral improvement was correlated with the reduction of activity in the M1 ROI (see above) during the illusion. In the series of fMRI results, we also reported the data obtained from whole-brain analysis. 


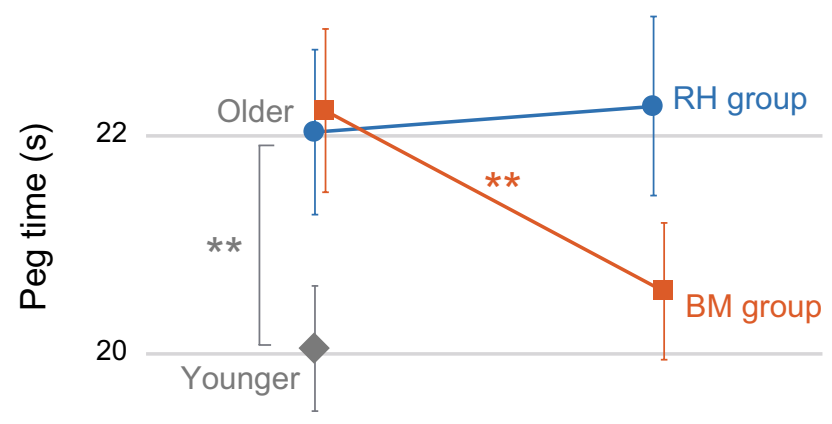

18

\section{Before After}

Figure 2. Peg time before (left) and after (right) the training. The orange squares indicate the average peg time in the BM group, the blue circles indicate that obtained in the RH group, and the gray diamond indicates that observed in the younger group. The error bars indicate the SEM. ${ }^{* *} P \leq 0.01$. BM bimanual, $R H$ right hand, SEM standard error of the mean.

\section{Results}

Before training. Peg task performance. All of the participants performed a 12-hole peg task ${ }^{6}$, during which the time required to flip all 12 pegs in each of three trials was measured. Although the average peg time was significantly longer in older adults than in younger adults $(22.13 \pm 3.65 \mathrm{~s}$ [mean \pm standard deviation] vs. $20.05 \pm 3.19 \mathrm{~s} ; t[77]=2.59, P=0.01$, effect size $\mathrm{d}=0.61$ ), no significant difference in peg time was observed between the two training groups of older adults $(\mathrm{BM}, 22.23 \pm 3.58 \mathrm{~s} ; \mathrm{RH}, 22.04 \pm 3.78 \mathrm{~s} ; t[46]=0.18, P=0.86$; Fig. 2 , left panel). This indicated that right-hand dexterity was deteriorated in older adults compared with younger adults, and that the pre-training peg time was highly similar between the two groups of older individuals.

Reduction/loss of ipsilateral deactivation in older adults. Task-related deactivation was examined during the illusion in the entire brain (family-wise error rate [FWE]-corrected extent threshold of $P<0.05$ across the entire brain for a voxel-cluster image generated at an uncorrected height threshold of $P<0.005$; blue sections in Fig. 3a). We found significant deactivation in the ipsilateral (right) SM1-PMD in the younger group, which was not observed in the older group (Fig. 3a; see Table S4a-d for other deactivations and activations).

The direct comparison of the activity between the two age groups (older vs. younger) revealed several significant clusters of voxels with between-age-group differences in the whole brain (pink section in Fig. 3a, bottom panel). The largest cluster was identified in the ipsilateral SM1-PMD and its peak was located in area $4 \mathrm{p}$ (see Table S5a for other clusters; see Table S5b for the opposite contrast [younger vs. older]). The ipsilateral SM1PMD cluster likely covered the hand/arm, trunk, face, and foot sections and extended into the foot section of the contralateral SM1.

We extracted the parameter estimate of brain activity from the ipsilateral M1 ROI (blue section in Fig. 3b) in each participant, and plotted it against their individual age. Almost all of the younger adults showed deactivation, whereas deactivation was generally reduced or lost in the older adults (Fig. 3b). This finding was corroborated by the analysis of the temporal profile of task-related brain activity during the illusion in a broader region of the hand/arm section of the bilateral SM1-PMD (see Supplementary Results and Fig. S1). However, no significant difference was observed between the two training (BM and RH) groups of older adults $\left(t^{46}=0.91, P=0.37\right.$; Fig. $3 \mathrm{~b}$ ). These results were observed even when the two age groups reported almost the same illusory flexion angle of the right-hand (older, $32.50^{\circ} \pm 15.98^{\circ}$; younger, $32.82^{\circ} \pm 13.37^{\circ} ; t[77]=0.09, P=0.93$; Table S6). In addition, the $\mathrm{BM}$ and $\mathrm{RH}$ groups reported almost the same illusory angle before training $(t[46]=1.20, P=0.23$; Table $S 6)$.

Correlation between deteriorated dexterity and reduction/loss of ipsilateral deactivation in older adults. Next, we examined whether the reduction/loss of ipsilateral M1 deactivation observed during the illusion is associated with the deterioration of dexterity in the older participants. We performed a regression analysis to depict brain regions in which deactivation was degraded with a longer peg time, i.e., a measure of deteriorated dexterity ${ }^{7,8}$. No significant clusters were found in the whole brain. However, as hypothesized, we found a significant cluster (pink section in Fig. 4; peak in area 4a; height threshold $P<0.005$ uncorrected) in the M1 ROI (blue and pink sections in Fig. 4) with small volume correction (SVC; 6 voxels, $P<0.05$ ). We also performed the same analysis in the young adults and found no significant clusters in the M1 ROI with SVC. Thus, the correlation between the 
a

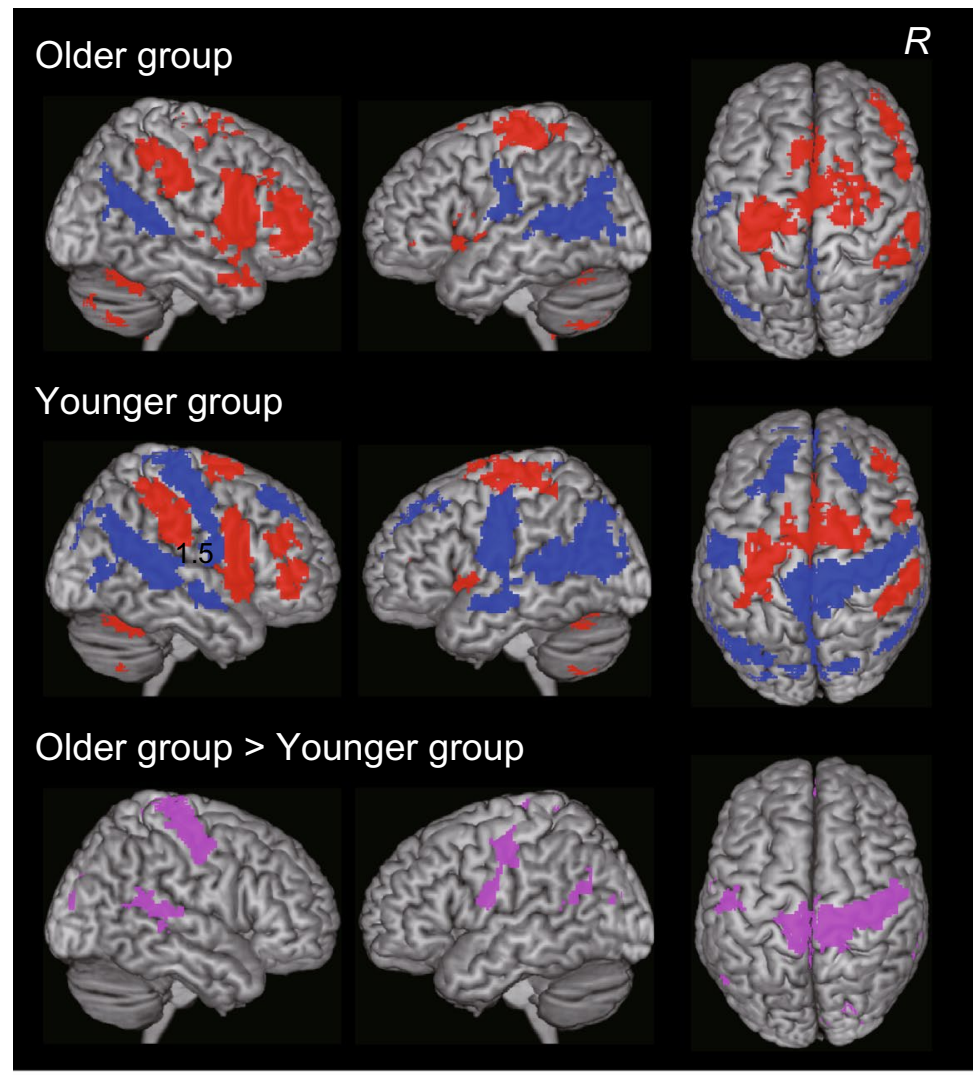

b

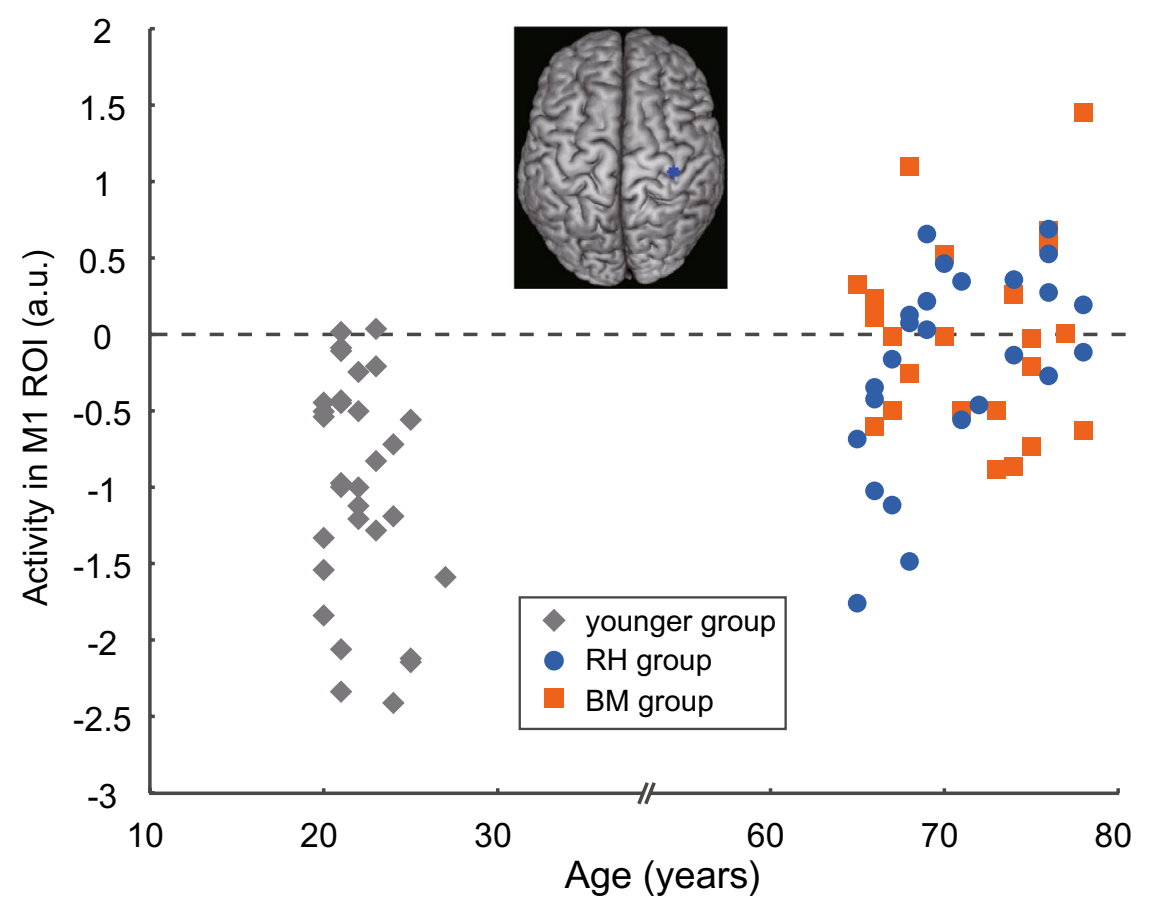

Figure 3. Brain deactivations and activations in the younger and older groups before training. (a) Brain deactivations (blue) and activations (red) observed during the illusion in the older (top) and younger (middle) groups. Table S4 summarizes the deactivations and activations. The pink areas indicate brain regions with greater activity in the older group than in the younger group (bottom). Table S5 summarizes the age-group difference results, which are rendered on the cortical surface of the MNI standard brain. In each row, the left panel indicates the right-side view, the middle panel indicates the left-side view, and the right panel indicates the top view of the brain. (b) Relationship between the parameter estimate of ipsilateral M1 ROI activity (a.u.; vertical axis) and age (horizontal axis). The blue section in the top panel indicates the ipsilateral M1 ROI. The data indicated below a dashed line indicate deactivation. The gray diamonds represent the data obtained from the younger group. The orange squares represent individual data obtained from the BM group. The blue circles indicate data from the RH group. a.u. arbitrary unit, BM bimanual, $M N I$ Montreal Neurological Institute, $R$ right hemisphere, $R H$ right hand. 


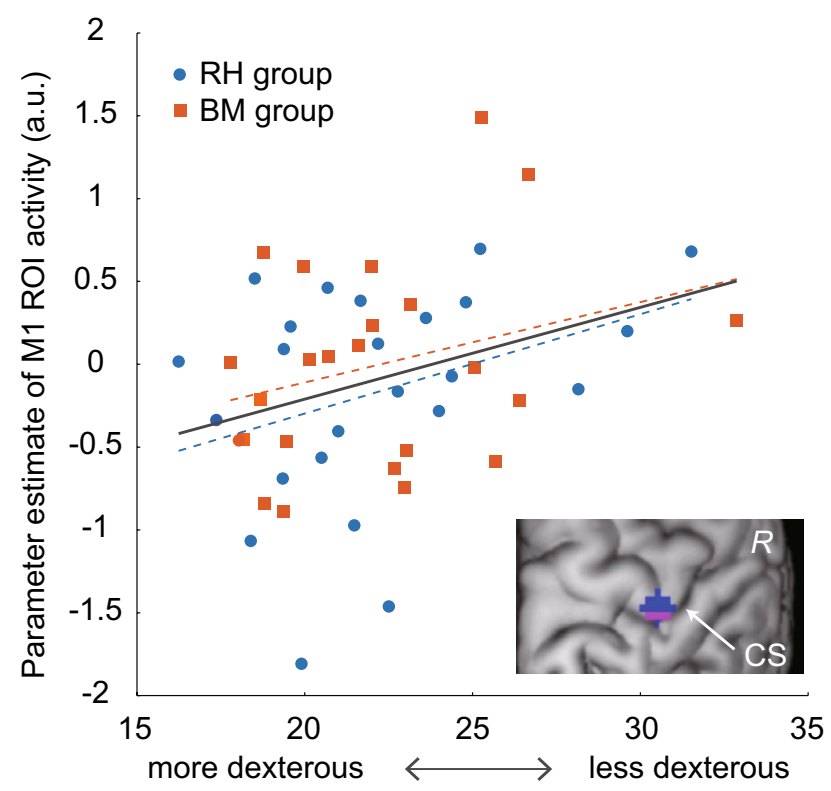

Peg time (s)

Figure 4. Relationship between the parameter estimate of ipsilateral M1 activity (a.u.; vertical axis) and the peg time (horizontal axis) across 48 older adults. The pink area in the right bottom panel indicates the ipsilateral M1 cluster in which the activity was positively correlated with the peg time. The parameter estimate was extracted from the M1 ROI (blue and pink areas in the right bottom panel). The orange squares represent individual data obtained from the BM group, and the orange dashed line indicates a regression line fitted to the data. The blue dots indicate individual data from the RH group, and the blue dashed line indicates a regression line fitted to the data. The grey solid line indicates a regression line fitted to all the data obtained from both groups. a.u. arbitrary unit, $B M$ bimanual, $C S$ central sulcus, $R$ right hemisphere, $R H$ right hand.

reduction/loss of deactivation in the focal region of the ipsilateral M1 (M1 ROI) and the deteriorated right-hand dexterity was only observed in the older group.

Such a positive correlation in the older group was confirmed by visualizing the relationship between the activity obtained from the M1 ROI and the peg time $(r=0.32, \mathrm{n}=48, P<0.05$; Fig. 4$)$. As shown in Fig. 4, in the older adults, a greater reduction or loss of ipsilateral M1 deactivation was associated with a greater deterioration of their right-hand dexterity. When we looked at the relationship in each group, we found similar regression lines (BM, $r=0.28, \mathrm{RH}, r=0.35$, Fig. 4). Indeed, no significant between-training-group differences were observed either in the slopes of the regression lines fitted to the data obtained from each group (BM or RH; F $[1,44]=0.054$, $P=0.82)$ or in their intercepts $(\mathrm{F}[1,45]=0.859, P=0.36)$, indicating that the two regression lines were not different. Thus, the results indicate that activity in the ipsilateral M1 region is related to dexterity deterioration in the older adults. The results reported above were observed in the absence of correlations between the illusory angle and the peg time $(r=0.11, \mathrm{n}=48, P=0.45)$ and between the angle and the ipsilateral M1 ROI activity $(r=0.15$, $\mathrm{n}=48, P=0.32)$ among the older participants.

After training. Peg task performance. We found no significant between-training-group differences in the number of training sets performed by each group (see Supplementary Methods and Table S3). Despite the absence of significant differences, we observed peg time improvement only in the BM group (Fig. 2). In this group, the peg time improved from $22.23 \pm 3.58 \mathrm{~s}$ (before) to $20.58 \pm 3.00 \mathrm{~s}$ (after). A two-way mixed-design analysis of variance (ANOVA; group [BM and $\mathrm{RH}] \times$ order [before and after]) of peg time showed a significant interaction between group and order $(\mathrm{F}[1,46]=7.67, P<0.01$, effect size $\mathrm{f}=0.14)$. A post hoc analysis revealed that the peg time became significantly shorter in the BM group after training $\left(t[22]=3.70\right.$, effect size $\mathrm{d}_{\mathrm{Z}}=0.77, P<0.005$ after Bonferroni correction), whereas no significant change was observed in the RH group $(t[24]=-0.46$, effect size $\mathrm{d}_{\mathrm{Z}}=0.09, P>1$ after Bonferroni correction). Thus, it was conceivable that the effect size of the peg time improvement in the BM group is large, and the improvement (1.65 s shorter on average) could be clinically meaningful change if we think that this time is practically equivalent to the time it takes to manipulate a single peg. These results showed that bimanual training, but not unimanual RH training, improved right hand/finger dexterity effectively.

Correlation between dexterity improvement and reduction of ipsilateral M1 activity. Because we found an improvement in dexterity only in the BM group, we performed a regression analysis to examine if the peg time improvement was associated with activity reduction after the training (=activity change after the training compared with before) in this group. We found a significant cluster in the hand/arm section of the ipsilateral 
a

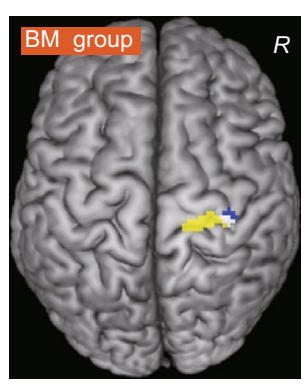

b

(a.u.)

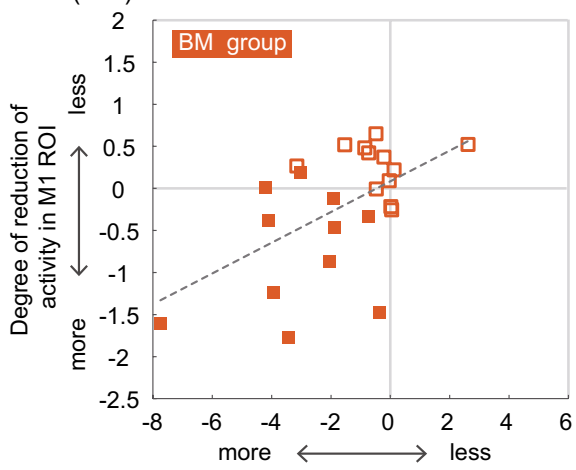

Degree of peg time improvement (s)
C

(a.u.)

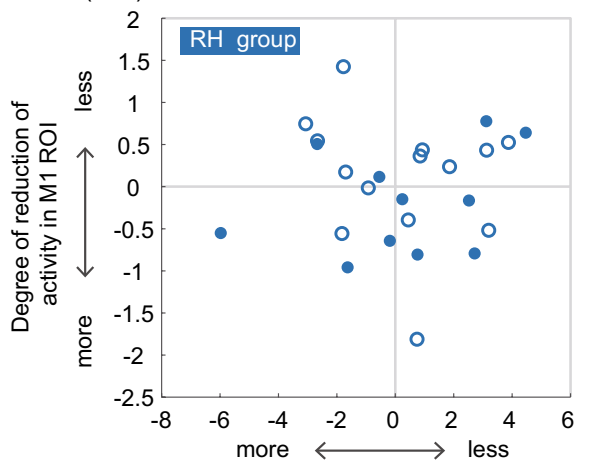

Degree of peg time improvement (s)

Figure 5. Relationship between the degree of reduction in ipsilateral M1 activity and peg time improvement. (a) Significant cluster (yellow and white areas) in which the degree of activity reduction was correlated with peg time improvement in the BM group. The cluster is superimposed on the MNI brain. (b,c) Relationship between the degree of reduction of activity (a.u.; vertical axis) in the M1 ROI (blue and white areas in panel a) and peg time improvement (s; horizontal axis) in each group (b for the BM group, $\mathbf{c}$ for the RH group). In each panel, the filled symbols represent data obtained from the participants who exhibited ipsilateral M1 hyperactivation (activity in the M1 ROI was greater than 0) before the training. The open symbols represent data obtained from the participants in whom ipsilateral M1 deactivation was preserved (activity in the M1 ROI was smaller than 0) before the training. The dashed line in panel (b) indicates a regression line fitted to the data. $a . u$. arbitrary unit, $B M$ bimanual, $M N I$ Montreal Neurological Institute, $R$ right hemisphere, $R H$ right hand.

SM1 (Fig. 5a), in addition to a cluster in the hand/arm sections of the bilateral caudal cingulate motor areas in the entire brain (see Table S7). Importantly, the ipsilateral SM1 cluster had peaks mainly in the M1 (Table S7) and overlapped with the M1 ROI (white section in Fig. 5a), in which we found a correlation between the degree of reduction/loss of deactivation and the deterioration of dexterity before training (blue and pink sections in Fig. 4). In contrast, in the RH group, we found no significant clusters with such a correlation in the entire brain.

Finally, we plotted the degree of reduction of activity (after-before) obtained from the M1 ROI against the peg time improvement (after-before) in each group, and confirmed the presence of a positive correlation in the BM group $(r=0.54, \mathrm{n}=23, P=0.008$; Fig. $5 \mathrm{~b})$. This implies that participants with a greater peg time improvement had a greater reduction of ipsilateral motor-cortical activity after the training. This correlation was observed even though there were no correlations between the change in illusory angle (after-before) and the peg time improvement $(r=0.12, \mathrm{n}=23, P=0.59)$ and between the change in angle and the degree of activity reduction $(r=0.26, \mathrm{n}=23, P=0.23)$ among the participants in the BM group. The $\mathrm{RH}$ group showed no such correlation $(r=0.03, \mathrm{n}=25, P=0.86$; Fig. $5 \mathrm{c})$.

Notably, we found, in the BM group, that the peg time improvement and the degree of reduction in the M1 ROI activity differed between participants who showed ipsilateral M1 hyperactivation (activity in the M1 ROI was greater than 0 ) during the illusion before the training and those who showed ipsilateral M1 deactivation (activity in the M1 ROI was smaller than 0 ). When we statistically evaluated between-subgroup differences, we found, in the BM group, that the peg time improvement and the degree of activity reduction were significantly greater in the participants with ipsilateral M1 hyperactivation $(n=11$; filled squares in Fig. $5 b)$ compared with those with ipsilateral deactivation $(\mathrm{n}=12$; open squares in Fig. $5 \mathrm{~b} ; t[21]=3.73$, effect size $\mathrm{d}=1.54, P<0.005$ for improvement, and $t[21]=4.52$, effect size $\mathrm{d}=1.86, P<0.001$ for reduction after Bonferroni correction). These findings were observed even when the number of training sets did not differ between these two subgroups $(t[21]=1.54, P=0.14)$. In contrast, no such differences were observed in the RH group $(t[23]=-0.04$, effect size $\mathrm{d}=0.01, P>1$ for improvement; $t[23]=1.04$, effect size $\mathrm{d}=0.43, P=0.62$ for reduction after Bonferroni correction; Fig. $5 c$ c). Hence, the bimanual training resulted in significantly greater behavioral and neuronal training effects in participants who had ipsilateral M1 activation (lost ipsilateral deactivation) before the training.

\section{Discussion}

This study clearly demonstrated that ipsilateral motor-cortical activity as an index of declined interhemispheric inhibition during a simple right-hand sensory-motor task was associated with deterioration of right hand/finger dexterity in older individuals. We also showed that bimanual digit training, which is available for anyone at any time and in any place, can improve unimanual dexterity by causing a reduction of ipsilateral motor-cortical activity especially in those who have ipsilateral motor-cortical hyperactivation before the training (Fig. 5b). These results revealed a tight relationship between interhemispheric inhibition and hand/finger dexterity in older adults, and suggest the trainability of the interhemispheric inhibitory system, which is deteriorated during normal aging, through bimanual training.

Physiological and technical considerations. In younger adults, ipsilateral SM1-PMD deactivation has been frequently reported during right-hand motor and proprioceptive tasks ${ }^{15-18}$. The physiological mechanisms 
underlying the task-induced negative BOLD phenomenon, which is indicative of deactivation, are not fully understood $^{19,20}$. However, many recent studies have suggested that this phenomenon is associated with neuronal inhibition (see the discussion in reference ${ }^{21}$ ), with no exception in the cerebro-cerebellar sensorimotor network $^{22,23}$.

The reduced interhemispheric inhibition exerted from the left M1 has been shown to be associated with a reduction/loss of deactivation in the right M1 in older adults ${ }^{10,24}$, and that the transcallosal interhemispheric inhibition exerted from the M1 to its opposite M1 can suppress activity in the latter ${ }^{25-28}$. Thus, the ipsilateral M1 deactivation observed during the right-hand illusion might be derived, at least partly, from interhemispheric inhibition exerted from the hand/arm section of the contralateral (left) M1, which was activated during the illusion (Fig. 3a), although we cannot fully exclude the possibility of inhibition from other brain structures, such as the thalamocortical system ${ }^{29}$, the $\mathrm{PMD}^{30,31}$ and $\mathrm{SMA}^{32}$.

In the current work, we evaluated ipsilateral sensorimotor deactivation (inhibition) using a proprioceptive task independent from the peg task, because such a complex finger task might activate the ipsilateral SM1-PMD, even in younger adults ${ }^{33}$, thus precluding the proper evaluation of the entity of ipsilateral inhibition in individual brains if brain activity is scanned using the peg task. In addition, ipsilateral SM1-PMD activity (i.e., reduction/ loss of deactivation) observed in the older adults during the non-motor task indicates that this phenomenon is not related to compensation of motor functions by the ipsilateral SM1-PMD (see below).

It has been suggested that, in older adults, a higher level of performance in a motor task is related to a higher capacity to modulate motor-cortical inhibition when performing the task $\mathrm{k}^{34}$, which largely depends on its basic state of local inhibition ${ }^{35}$. We assumed that the ipsilateral SM1-PMD deactivation observed during the illusion may reflect the "basic state" of its local inhibition, because this is likely caused mainly by the interhemispheric inhibition exerted from the contralateral SM1-PMD when this region is activated by simply receiving a kinesthetic input. Therefore, the reduction/loss of ipsilateral SM1-PMD deactivation (i.e., basic state) observed during the illusion might be associated with a reduced capability to modulate ipsilateral motor-cortical inhibition when the older participants performed the peg task; however, the confirmation of this claim should be the subject of future studies.

Deteriorated dexterity and its relation to ipsilateral activity in older adults before training. The degree of reduction/loss of ipsilateral M1 deactivation was correlated with deteriorated dexterity in the older group (Fig. 4). This finding was confirmed by the analysis performed using a larger sample of older participants (see Supplementary Results and Fig. S2). Thus, these findings are consistent with those reported previously ${ }^{7,8}$. Notably, the M1 region is identical to the region that was identified in our previous study, which showed that the degree of deactivation was associated with a better right hand/finger dexterity during childhood ${ }^{6}$. Interestingly, such a correlation was not observed in the younger group, as demonstrated in our previous study ${ }^{6}$. In another study, we also demonstrated that ipsilateral M1 deactivation during a right-hand unimanual motor task progresses from childhood to adolescence, but stabilizes from adolescence to adulthood ${ }^{21}$, indicating that the functional differentiation between the left and right M1 occurs between childhood and adolescence. Therefore, ipsilateral M1 deactivation might be deeply associated with hand/finger dexterity during childhood, when the deactivation (inhibition) is developing, and during elderhood, when it deteriorates. These findings suggest that ipsilateral M1 inhibition in the central motor system substantially influences dexterous motor control during childhood and old age.

In nonhuman primates, motor neurons in lamina IX of the spinal cord directly receive efferents primarily from the contralateral M1. This indicates that the fast, direct corticomotor pathway, which enables fine, dexterous finger movements ${ }^{4,5}$, originates primarily from the contralateral M1, particularly the new M1 (the caudal region of the M1) $)^{36}$, which likely corresponds to the human area $4 \mathrm{p}^{37}$. Although the ipsilateral M1 also projects to the spinal cord, there are fewer terminals in lamina $\mathrm{IX}^{38}$, indicating that the ipsilateral M1 projection is not suitable for the direct, fine control of hand/finger muscles; rather, it is suitable for muscle synergy control. The development of ipsilateral M1 deactivation that occurs from childhood to adolescence ${ }^{21}$, together with the development of transcallosal interhemispheric inhibition during these periods ${ }^{39}$, suggests that the brain develops to facilitate dominance of the contralateral M1 for the fine control of dexterous hand/finger movements by inhibiting the ipsilateral M1, although the brain is capable of recruiting the ipsilateral M1 in cases of brain stroke ${ }^{40-42}$. We assumed that the capability to inhibit the ipsilateral M1 allows the brain to avoid potential disturbance from the M1, for elaborate control by the contralateral one $e^{6}$. This view seems to be compatible with our findings that ipsilateral M1 activity was correlated with deteriorated dexterity (Fig. 4) and that the reduction of ipsilateral M1 activity was associated with dexterity improvement (Fig. 5) in older adults, who may partially utilize the ipsilateral M1 when performing a motor task ${ }^{43}$.

We assumed that a possible decline in interhemispheric inhibitory function, which can suppress and regulate ipsilateral M1 activity (see above), is one of the factors that contribute to the reduction/loss of ipsilateral M1 deactivation (inhibition) in older adults. Although little is known about the exact neuronal mechanisms underlying decreased interhemispheric inhibition, the quantitative and qualitative deterioration of transcallosal nerve fibers observed in older adults could be related to this decline ${ }^{13,44-46}$. Moreover, a reduction in the level of the inhibitory neurotransmitter gamma-aminobutyric acid (GABA) in the frontoparietal cortices of older adults could also be related to decreased inhibition ${ }^{47}$.

If we consider the relationship discovered here between the reduction/loss of ipsilateral M1 deactivation and the deteriorated dexterity observed in the healthy older adults (Fig. 4) together with the fact that patients with stroke who lack dexterity often exhibit ipsilateral SM1-PMD activity, we may assume that ipsilateral SM1-PMD activity is an index of the deterioration of dexterity in these individuals. This view does not contradict the fact that the compensatory ipsilateral SM1-PMD activity that occurs after contralateral SM1-PMD stroke often disappears 
when hand/finger dexterity improves ${ }^{41}$. The reduction/loss of ipsilateral M1 deactivation detected during the illusion in the older adults (Fig. 3b) likely represents a chronic decline in ipsilateral M1 inhibition. As described above, ipsilateral SM1-PMD activity is often reported when people perform complex finger movements ${ }^{33}$ and precision-demanding tasks ${ }^{48,49}$. This could be caused by an "active" disinhibition, in which the brain utilizes the ipsilateral SM1-PMD by affirmatively releasing interhemispheric inhibition; this could be distinct from the chronic decline observed in older adults. In turn, this decline in older adults might indicate a lesser potential of their brains to disinhibit the ipsilateral M1 (i.e., compensation by the ipsilateral M1) in cases of stroke.

Improvement of right-hand dexterity and its relation to reduced ipsilateral M1 activity after training. Bimanual training improved right-hand dexterity (Fig. 2) in association with reduced ipsilateral M1 activity during the illusion (Fig. 5b). Because the present training did not include the peg task training per se, we attributed this behavioral improvement to non-peg-task-specific neuronal changes caused by the bimanual training, i.e., most likely the reactivation of interhemispheric inhibition. Importantly, the bimanual training resulted in significantly greater behavioral and neuronal training effects in participants who exhibited ipsilateral hyperactivation before the training (Fig. 5b), clearly indicating the effectiveness of bimanual training for older adults who need reactivation of ipsilateral inhibition.

The claim that bimanual training reactivates interhemispheric inhibition seems to be supported by the following evidence. The analysis of functional connectivity during the illusion revealed that the bimanual training reduced the functional connectivity between the bilateral SM1-PMDs compared with the RH training (see Supplementary Results, Fig. S3, and Table S8). In the present study, no significant correlation was observed between the reduction of interhemispheric functional connectivity and dexterity improvement. However, a previous study reported that the greater functional connectivity detected between the left and right M1 regions in older adults, which might reflect an aging-related release from the normally predominantly inhibitory interhemispheric communication, was associated with poorer performance in an unimanual finger tapping task ${ }^{12}$. The decrease in interhemispheric connectivity observed here after the bimanual training suggests that the brain changed to afford the activation of the two SM1-PMDs independently of each other. This suggests that the transcallosal interhemispheric inhibitory system is reactivated after the training to suppress a more synchronized activity between the two SM1-PMDs before the training. Thus, the various findings reported here indicate that bimanual training may reactivate the interhemispheric inhibitory system between the bilateral SM1-PMDs more effectively than unimanual RH training. However, the lack of electrophysiological measurement of interhemispheric inhibition using transcranial magnetic stimulation was one of the limitations of the present study. Therefore, further studies are needed to support our claim. However, if we consider the study that reported that 12 -week aerobic whole-body exercises improved interhemispheric inhibition and hand/finger dexterity in older adults ${ }^{50}$, then the interhemispheric inhibitory system, which deteriorates with normal aging, can be trained.

Theoretically, the RH training could also lead to an inhibitory effect on the ipsilateral M1, because it has been shown that, in younger adults, the exclusive use of one hand elevates the motor-cortical excitability in the contralateral M1, which leads to a stronger inhibitory effect on the ipsilateral M1 ${ }^{51}$. However, our results suggest that, in older adults in whom transcallosal interhemispheric inhibition is likely deteriorated ${ }^{7,12}$, unimanual training might not effectively decrease ipsilateral M1 activity, probably because of the coactive mode between the bilateral SM1-PMDs (see Fig. S1).

Ipsilateral M1 activity and hemispheric asymmetry reduction in older adults (HAROLD). Our finding that ipsilateral M1 activity, as an index of declined interhemispheric inhibition, was associated with the deterioration of right-hand dexterity in older adults was compatible with previous reports ${ }^{7-9,50}$. This was a finding when we measured the activity during the illusion when the participants were motionless and had no intention to move the right-hand. On the other hand, there is a finding that a faster reaction time in a simple button-press task was correlated with greater ipsilateral premotor activity during the task in older adults ${ }^{52}$. It remains controversial whether additional activation and overactivation in older adults reflect compensatory recruitment or reorganization ${ }^{1}$ or are simply a consequence of an age-related decline in the inhibitory process ${ }^{53}$. For example, it seems that the additional activations in the left frontoparietal cortices (but not in the ipsilateral SM1-PMD) that occur during a right-hand-foot coordination task can be considered as the former ${ }^{54}$, whereas declined interhemispheric inhibition can be considered as the latter. The latter view seems to also be supported by reports that motor overflow (involuntary movement or muscle activity) in passive homologous muscles contralateral to voluntary movement is often greater in older adults vs. younger adults, possibly because of a decline in interhemispheric inhibition in older adults ${ }^{55}$.

Prefrontal activity during cognitive (e.g., memory) tasks tends to be less lateralized (i.e., showing bilateral activity) in older adults vs. younger adults. This phenomenon is called hemispheric asymmetry reduction in older adults (HAROLD) ${ }^{56}$. The reduction/loss of ipsilateral SM1-PMD deactivation observed during the illusion in older adults seems to be a type of HAROLD (Fig. 3b). The present sensorimotor HAROLD (i.e., reduction/ loss of ipsilateral M1 deactivation) was associated with deteriorated dexterity (Fig. 4; see also references ${ }^{7-9,50}$ ), whereas prefrontal HAROLD may have a compensatory function for maintaining cognitive performance ${ }^{57}$. A study of resting-state functional connectivity has suggested that cross-hemispheric connectivity is higher in the sensorimotor cortex, whereas within-hemispheric connectivity is higher in the prefrontal cortex, in younger adults $^{58}$. Thus, interhemispheric inhibition between two sensorimotor cortices might be necessary for the brain to utilize each hemisphere independently against their intrinsically synchronous mode. Hence, we raise the possibility that HAROLD in the bilateral primary sensorimotor cortices can be observed as a mere result of the agerelated decline of interhemispheric inhibition, as if the system becomes infantilized, although the sensorimotor HAROLD may become beneficial when the brain generates symmetrical bimanual movements by recruiting the 
bilateral sensorimotor cortices in a coactive mode (see also reference ${ }^{55}$ ), as well as in cases that require a faster reaction that does not require dexterity ${ }^{52}$.

The brain of older individuals can be characterized by a reduction/loss of various interregional brain deactivations (inhibition) during cognitive and sensorimotor tasks ${ }^{59-62}$. The current work suggests that training tasks that can facilitate interregional brain communications might improve the cognitive and sensorimotor functions that are deteriorated in older individuals by reactivating their declined interregional inhibitory system that prevails in younger adults.

\section{Methods}

Participants. The current work was a single-blinded, randomized, controlled study (Fig. 1 and Supplementary Methods). We first recruited 53 older adults (65-78 years). The sample size was determined by a power analysis using $\mathrm{G}^{*}$ power ver. 3.1.9.7 (https:/www.psychologie.hhu.de/arbeitsgruppen/allgemeine-psychologieund-arbeitspsychologie/gpower.html) for the paired t-test (two-tailed, effect size $=0.6, \alpha=0.05, \beta=0.80$ ). The power analysis provides the sample size of $n=24$. Considering the necessity of having two training groups and the possibility of dropouts during the two-month training, we decided to recruit 53 older adults. Our inclusion criteria of participants were healthy, right-handed older adults with no history of neurological, psychiatric, or movement disorders. One person was excluded because he did not meet the criteria. The 52 older participants were divided into two training groups: the BM group $(n=26)$ and the RH group $(n=26)$. During two-month training, two participants in the BM group and one participant in the RH group were dropped out. In the analysis, we excluded one participant in the BM group due to his deviated data. Eventually, we analyzed the data obtained from 23 older participants in the BM group (age, $71.7 \pm 4.3$ [mean \pm standard deviation] years; males, 14 ) and from 25 older participants in the RH group (age, $70.6 \pm 4.2$ years; males, 17). We assessed the cognitive status of older participants using the Mini-Mental State Examination. All participants scored higher than the cut-off score of $24^{63}$, and no significant difference was observed between the RH (28.4 \pm 1.9$)$ and BM (28.8 \pm 1.6$)$ groups. We also recruited healthy, right-handed younger adults with no history of neurological, psychiatric, or movement disorders $(n=31$; age, $22.1 \pm 1.8$ years; age range, $20-27$ years; males, 22$)$. We confirmed the handedness of the participants using the Edinburgh Handedness Inventory ${ }^{64}$.

The study protocol was approved by the Ethics Committee of the National Institute of Information and Communications Technology. We explained the details of the present study to all participants before the experiment, and they then provided written informed consent. The study was conducted according to the principles and guidelines of the Declaration of Helsinki (1975).

Peg task to evaluate hand/finger dexterity. We used a 12-hole peg task to evaluate right-hand/finger dexterity $^{6}$, because peg tasks have generally been used to evaluate hand/finger dexterity, particularly that of the fingertips, across individuals with a wide age range $\mathrm{e}^{7-9}$. Participants had to remove a small peg that had been inserted in one of 12 holes on a board using their right fingers, vertically flip the peg, and reinsert the peg into the same hole, repeatedly. We measured the time required to flip all 12 pegs using a stopwatch. Because none of our participants had previously experienced this task, each participant performed the peg task three times. In this task, a participant who is better able to coordinate her/his fingertip movements rapidly, without generating superfluous movements, should be able to complete the task more quickly.

Before training, we calculated the average time (peg time) of three trials in the older and younger groups, respectively. We evaluated the between-age-group differences by conducting a two-sample $t$-test. To evaluate the training effect on peg task performance, we also calculated the peg time for the two training groups after training. We conducted an ANOVA that included one between-subject factor (group [2]: BM or RH) and one within-subject factor (order [2]: before and after), and further performed a post hoc test with Bonferroni correction to evaluate the possible training effects in each group. In these analyses, we calculated effect size using $\mathrm{G}^{\star}$ power ver. 3.1.9.7 (see above).

Two-month training. Only the older participants performed approximately two months of daily training. For each training group, we prepared five types of training menus; the details of the menus performed by each group, the training procedure, and the mean number of sets of training menus performed during the training period are provided in the Supplementary Methods and Tables S1, S2 and S3. The participants had to perform these menus at home every day (homework) until the day before the second MRI day.

fMRI task. Kinesthetic illusion task. A kinesthetic illusion task was used to evaluate the ipsilateral SM1PMD deactivation, as described previously ${ }^{65}$. Briefly, we vibrated the tendon of the extensor carpi ulnaris muscle of the relaxed right wrist, which elicited an illusory flexion of the stationary right-hand ${ }^{11}$. Detail of this task procedure is described in the Supplementary Methods.

One run consisted of five tendon-vibration epochs (each lasting for $15 \mathrm{~s}$ ). The tendon-vibration epochs were separated by 15-s baseline periods. During the baseline period, we vibrated the skin surface over a nearby bone (i.e., the processus styloideus ulnae of the hand next to the tendon; bone vibration) using the same stimulus, which mainly elicits a cutaneous vibration sensation with no reliable illusion, to control skin vibration and attentional effects ${ }^{65}$. Each run also included a 25-s period before the start of the first epoch.

During fMRI scanning, we asked the participants to close their eyes, relax their entire body, refrain from producing unnecessary movements, and be aware of movement sensations from the vibrated hand (during both tendon vibration and bone vibration). After each experimental run, we measured maximum illusory angle that they experienced in a run (see detail in Supplementary Methods), and the mean illusory angle between two runs 
was calculated for each participant. Detail of statistical analyses are described in the Supplementary Methods. The illusory angles recorded in each group are summarized in Table S6.

Single-subject analysis. Details of MRI data acquisition and image preprocessing are described in the Supplementary Methods. After the conventional preprocessing of MRI data, we used a general linear model ${ }^{66}$ to analyze fMRI data. We prepared a design matrix for each participant to analyze the functional images before training. The design matrix contained a boxcar function for the task epoch in each run, which was convolved with a canonical hemodynamic response function. To correct for residual motion-related variance after realignment, six realignment parameters were also included in the design matrix as regressors of no interest. In the analysis, we did not perform global mean scaling to avoid inducing type I errors in the assessment of negative BOLD responses ${ }^{67}$. We generated an image showing task-related activation/deactivation in each participant. For older adults, we generated another contrast image showing the difference between the results obtained before and after training (after-before) in each older participant. These images were used in the subsequent second-level group analyses.

Analyses before training. We examined task-related activation/deactivation during the illusion using a secondlevel group analysis ${ }^{68}$ with a one-sample $t$-test to illustrate the patterns of task-related activation/deactivation across the entire brain in each age group (Fig. 3a). We also examined between-age-group differences by comparing the older group with the younger group (older vs. younger). We used a family-wise error rate [FWE]corrected extent threshold of $P<0.05$ across the entire brain for a voxel-cluster image generated at the uncorrected height threshold of $P<0.005$, which was consistently used in the present whole-brain analyses. For the anatomical definition of the identified peaks, we referred to the cytoarchitectonic map implemented in the SPM Anatomy toolbox ${ }^{69}$, which was also consistently used in the present study. To confirm the between-age-group differences in the ipsilateral (right) M1 region, we set an ipsilateral M1 ROI as a 4-mm radius sphere around the M1 peak $(36,-26,66)$, in which the degree of deactivation is correlated with a better right hand/finger dexterity during childhood ${ }^{6}$. We extracted the parameter estimate of brain activity from the M1 ROI for each participant and plotted it against their individual ages (Fig. 3b).

We performed a regression analysis to test whether the degree of reduction/loss of ipsilateral M1 deactivation is associated with dexterity deterioration in the older group $(\mathrm{n}=48)$. In this analysis, we hypothesized that a region with such a correlation could be observed in the M1 ROI, in which the degree of deactivation is correlated with a better right hand/finger dexterity during childhood ${ }^{6}$. Based on this strong anatomical hypothesis, we applied an SVC $(P<0.05)^{70}$ with the M1 ROI (above). Finally, to illustrate the relationship between the individual degree of ipsilateral M1 activity and the individual peg time in the older group, we extracted the data from the M1 ROI and plotted them against the peg time (Fig. 4). We fitted a regression line to the data obtained from each group (BM and $\mathrm{RH}$ ). We used an analysis of covariance for the statistical evaluation of the slopes of the regression lines and their intercepts.

Regression analysis between peg time improvement and brain activity change. Because we found that the dexterity improved in the BM group exclusively, we further conducted a regression analysis to examine brain regions in which the change in activity observed after the training was associated with the improvement in peg time in this group. To define the individual peg time change, we subtracted the peg time before the training from that after the training (after-before) for each participant. We used the individual contrast image (after-before) to identify brain regions in which the activity change was correlated with the individual peg time change in the entire brain (Fig. 5a). The same analysis was performed for the RH group. To verify the correlation observed in the BM group, we plotted the individual degree of reduction in ipsilateral M1 activity obtained from the M1 ROI (see above) against the peg time improvement in each group (Fig. 5b,c).

Finally, because we found that the peg time improvement and the degree of reduction in ipsilateral M1 ROI activity in the BM group were greater in participants $(n=11)$ with ipsilateral hyperactivation in the M1 ROI (activity was greater than 0$)$ during the illusion before the training than in those $(n=12)$ with ipsilateral deactivation (activity was smaller than 0 ), we conducted a post hoc two-sample $t$-test with Bonferroni correction to evaluate possible between-subgroup differences. We performed the same post hoc analysis for the RH group. In these analyses, we also reported effect sizes using $G^{*}$ power ver. 3.1.9.7 (see above).

\section{Data availability}

The datasets generated during and/or analyzed during the current study are available from the corresponding author on reasonable request.

Received: 27 May 2021; Accepted: 8 November 2021

Published online: 22 November 2021

\section{References}

1. Ward, N. S. Compensatory mechanisms in the aging motor system. Ageing Res. Rev. 5, 239-254 (2006).

2. Seidler, R. D. et al. Motor control and aging: Links to age-related brain structural, functional, and biochemical effects. Neurosci. Biobehav. Rev. 34, 721-733 (2010).

3. Heffner, R. S. \& Masterton, R. B. The role of the corticospinal tract in the evolution of human digital dexterity. Brain. Behav. Evol. 23, 165-183 (1983).

4. Isa, T. Dexterous hand movements and their recovery after central nervous system injury. Annu. Rev. Neurosci. 42, 315-335 (2019).

5. Lemon, R. Recent advances in our understanding of the primate corticospinal system. F1000Research 8 (2019). 
6. Naito, E., Morita, T. \& Asada, M. Importance of the primary motor cortex in development of human hand/finger dexterity. Cereb. Cortex Commun. 1, 085 (2020).

7. Davidson, T. \& Tremblay, F. Age and hemispheric differences in transcallosal inhibition between motor cortices: An ispsilateral silent period study. BMC Neurosci. 14, 62 (2013).

8. Loibl, M., Beutling, W., Kaza, E. \& Lotze, M. Non-effective increase of fMRI-activation for motor performance in elder individuals. Behav. Brain Res. 223, 280-286 (2011).

9. McGregor, K. M. et al. Aging, aerobic activity and interhemispheric communication. Brain Sci. 2, 634-648 (2012).

10. Talelli, P., Ewas, A., Waddingham, W., Rothwell, J. C. \& Ward, N. S. Neural correlates of age-related changes in cortical neurophysiology. Neuroimage 40, 1772-1781 (2008).

11. Naito, E., Morita, T. \& Amemiya, K. Body representations in the human brain revealed by kinesthetic illusions and their essential contributions to motor control and corporeal awareness. Neurosci. Res. 104, 16-30 (2016).

12. Fling, B. W. et al. Differential callosal contributions to bimanual control in young and older adults. J. Cogn. Neurosci. 23, 2171-2185 (2011).

13. Fling, B. W. \& Seidler, R. D. Fundamental differences in callosal structure, neurophysiologic function, and bimanual control in young and older adults. Cereb. Cortex 22, 2643-2652 (2012).

14. Kelso, J. A. Phase transitions and critical behavior in human bimanual coordination. Am. J. Physiol.-Regul. Integr. Comp. Physiol. 246, R1000-R1004 (1984).

15. Allison, J. D., Meador, K. J., Loring, D. W., Figueroa, R. E. \& Wright, J. C. Functional MRI cerebral activation and deactivation during finger movement. Neurology 54, 135-142 (2000).

16. Newton, J. M., Sunderland, A. \& Gowland, P. A. fMRI signal decreases in ipsilateral primary motor cortex during unilateral hand movements are related to duration and side of movement. Neuroimage 24, 1080-1087 (2005).

17. Hayashi, M. J. et al. Hemispheric asymmetry of frequency-dependent suppression in the ipsilateral primary motor cortex during finger movement: A functional magnetic resonance imaging study. Cereb. Cortex 18, 2932-2940 (2008).

18. Petsas, N. et al. Evidence of impaired brain activity balance after passive sensorimotor stimulation in multiple sclerosis. PLoS ONE 8, e65315 (2013).

19. Kim, S.-G. \& Ogawa, S. Biophysical and physiological origins of blood oxygenation level-dependent fMRI signals. J. Cereb. Blood Flow Metab. 32, 1188-1206 (2012).

20. Moraschi, M., DiNuzzo, M. \& Giove, F. On the origin of sustained negative BOLD response. J. Neurophysiol. 108, 2339-2342 (2012).

21. Morita, T., Asada, M. \& Naito, E. Developmental changes in task-induced brain deactivation in humans revealed by a motor task. Dev. Neurobiol. 79, 536-558 (2019).

22. Hummel, F. et al. To act or not to act. Neural correlates of executive control of learned motor behavior. Neuroimage 23, 1391-1401 (2004).

23. Mullinger, K. J., Mayhew, S. D., Bagshaw, A. P., Bowtell, R. \& Francis, S. T. Evidence that the negative BOLD response is neuronal in origin: A simultaneous EEG-BOLD-CBF study in humans. Neuroimage 94, 263-274 (2014).

24. McGregor, K. M. et al. Physical activity and neural correlates of aging: A combined TMS/fMRI study. Behav. Brain Res. 222, 158-168 (2011).

25. Ferbert, A. et al. Interhemispheric inhibition of the human motor cortex. J. Physiol. 453, 525-546 (1992).

26. Kobayashi, M., Hutchinson, S., Schlaug, G. \& Pascual-Leone, A. Ipsilateral motor cortex activation on functional magnetic resonance imaging during unilateral hand movements is related to interhemispheric interactions. Neuroimage 20, 2259-2270 (2003).

27. Hübers, A., Orekhov, Y. \& Ziemann, U. Interhemispheric motor inhibition: Its role in controlling electromyographic mirror activity. Eur. J. Neurosci. 28, 364-371 (2008).

28. Uehara, K. \& Funase, K. Contribution of ipsilateral primary motor cortex activity to the execution of voluntary movements in humans: A review of recent studies. J. Phys. Fit. Sports Med. 3, 297-306 (2014).

29. Blankenburg, F. et al. Imperceptible stimuli and sensory processing impediment. Science 299, 1864 (2003).

30. Uehara, K., Morishita, T., Kubota, S. \& Funase, K. Neural mechanisms underlying the changes in ipsilateral primary motor cortex excitability during unilateral rhythmic muscle contraction. Behav. Brain Res. 240, 33-45 (2013).

31. Reis, J. et al. Contribution of transcranial magnetic stimulation to the understanding of cortical mechanisms involved in motor control. J. Physiol. 586, 325-351 (2008)

32. Volz, L. J., Eickhoff, S. B., Pool, E.-M., Fink, G. R. \& Grefkes, C. Differential modulation of motor network connectivity during movements of the upper and lower limbs. Neuroimage 119, 44-53 (2015).

33. Uehara, S., Nambu, I., Matsumura, M., Kakei, S. \& Naito, E. Prior somatic stimulation improves performance of acquired motor skill by facilitating functional connectivity in cortico-subcortical motor circuits. J. Behav. Brain Sci. 2, 343-356 (2012).

34. Levin, O., Fujiyama, H., Boisgontier, M. P., Swinnen, S. P. \& Summers, J. J. Aging and motor inhibition: A converging perspective provided by brain stimulation and imaging approaches. Neurosci. Biobehav. Rev. 43, 100-117 (2014).

35. Heise, K.-F. et al. The aging motor system as a model for plastic changes of GABA-mediated intracortical inhibition and their behavioral relevance. J. Neurosci. 33, 9039-9049 (2013).

36. Rathelot, J.-A. \& Strick, P. L. Subdivisions of primary motor cortex based on cortico-motoneuronal cells. Proc. Natl. Acad. Sci. 106, 918-923 (2009).

37. Geyer, S. et al. Two different areas within the primary motor cortex of man. Nature 382, 805-807 (1996).

38. Morecraft, R. J. et al. Terminal distribution of the corticospinal projection from the hand/arm region of the primary motor cortex to the cervical enlargement in rhesus monkey. J. Comp. Neurol. 521, 4205-4235 (2013).

39. Ciechanski, P., Zewdie, E. \& Kirton, A. Developmental profile of motor cortex transcallosal inhibition in children and adolescents. J. Neurophysiol. 118, 140-148 (2017).

40. Willer, C., Ramsay, S. C., Wise, R. J. S., Friston, K. J. \& Frackwiak, R. S. J. Individual patterns of functional reorganization in the human cerebral cortex after capsular infraction. Ann. Neurol. 33, 181-189 (1993).

41. Jang, S. H. et al. Cortical activation changes associated with motor recovery in patients with precentral knob infarct. NeuroReport 15, 395-399 (2004).

42. Lotze, M. et al. The role of multiple contralesional motor areas for complex hand movements after internal capsular lesion. J. Neurosci. Off. J. Soc. Neurosci. 26, 6096-6102 (2006).

43. Fujiyama, H., Hinder, M. R. \& Summers, J. J. Functional role of left PMd and left M1 during preparation and execution of left hand movements in older adults. J. Neurophysiol. 110, 1062-1069 (2013).

44. Sullivan, E. V., Adalsteinsson, E. \& Pfefferbaum, A. Selective age-related degradation of anterior callosal fiber bundles quantified in vivo with fiber tracking. Cereb. Cortex N. Y. N 1991(16), 1030-1039 (2006).

45. Ota, M. et al. Age-related degeneration of corpus callosum measured with diffusion tensor imaging. Neuroimage 31, 1445-1452 (2006).

46. Lebel, C. et al. Diffusion tensor imaging of white matter tract evolution over the lifespan. Neuroimage 60, 340-352 (2012).

47. Gao, F. et al. Edited magnetic resonance spectroscopy detects an age-related decline in brain GABA levels. Neuroimage 78, 75-82 (2013).

48. Buetefisch, C. M., Revill, K. P., Shuster, L., Hines, B. \& Parsons, M. Motor demand-dependent activation of ipsilateral motor cortex. J. Neurophysiol. 112, 999-1009 (2014). 
49. Barany, D. A. et al. Primary motor cortical activity during unimanual movements with increasing demand on precision. J. Neurophysiol. 124, 728-739 (2020).

50. McGregor, K. M. et al. Influences of 12-week physical activity interventions on TMS measures of cortical network inhibition and upper extremity motor performance in older adults-A feasibility study. Front. Aging Neurosci. 9, 422 (2017).

51. Avanzino, L., Bassolino, M., Pozzo, T. \& Bove, M. Use-dependent hemispheric balance. J. Neurosci. 31, 3423-3428 (2011).

52. Mattay, V. S. et al. Neurophysiological correlates of age-related changes in human motor function. Neurology 58, 630-635 (2002).

53. Fling, B. W., Kwak, Y., Peltier, S. J. \& Seidler, R. D. Differential relationships between transcallosal structural and functional connectivity in young and older adults. Neurobiol. Aging 33, 2521-2526 (2012).

54. Heuninckx, S., Wenderoth, N. \& Swinnen, S. P. Systems neuroplasticity in the aging brain: Recruiting additional neural resources for successful motor performance in elderly persons. J. Neurosci. 28, 91-99 (2008).

55. Addamo, P. K., Farrow, M., Hoy, K. E., Bradshaw, J. L. \& Georgiou-Karistianis, N. The effects of age and attention on motor overflow production-A review. Brain Res. Rev. 54, 189-204 (2007).

56. Cabeza, R. Hemispheric asymmetry reduction in older adults: the HAROLD model. Psychol. Aging 17, 85-100 (2002).

57. Cabeza, R. et al. Maintenance, reserve and compensation: The cognitive neuroscience of healthy ageing. Nat. Rev. Neurosci. 19, 701-710 (2018).

58. Wang, D., Buckner, R. L. \& Liu, H. Functional specialization in the human brain estimated by intrinsic hemispheric interaction. J. Neurosci. 34, 12341-12352 (2014).

59. Riecker, A. et al. Functional significance of age-related differences in motor activation patterns. Neuroimage 32, 1345-1354 (2006).

60. Ward, N. S., Swayne, O. B. C. \& Newton, J. M. Age-dependent changes in the neural correlates of force modulation: An fMRI study. Neurobiol. Aging 29, 1434-1446 (2008).

61. Lustig, C. et al. Functional deactivations: Change with age and dementia of the Alzheimer type. Proc. Natl. Acad. Sci. 100, 1450414509 (2003).

62. Morita, T., Asada, M. \& Naito, E. Examination of the development and aging of brain deactivation using a unimanual motor task. Adv. Robot. 10, 1-16 (2021).

63. Lopez, M. N., Charter, R. A., Mostafavi, B., Nibut, L. P. \& Smith, W. E. Psychometric properties of the Folstein Mini-Mental State Examination. Assessment 12, 137-144 (2005).

64. Oldfield, R. C. The assessment and analysis of handedness: The Edinburgh inventory. Neuropsychologia 9, 97-113 (1971).

65. Morita, T., Asada, M. \& Naito, E. Right-hemispheric dominance in self-body recognition is altered in left-handed individuals. Neuroscience 425, 68-89 (2020).

66. Worsley, K. J. \& Friston, K. J. Analysis of fMRI time-series revisited-again. Neuroimage 2, 173-181 (1995).

67. Aguirre, G. K., Zarahn, E. \& D’Esposito, M. The inferential impact of global signal covariates in functional neuroimaging analyses. Neuroimage 8, 302-306 (1998).

68. Holmes, A. P. \& Friston, K. J. Generalisability, random effects and population inference. Neuroimage 7, S754 (1998).

69. Eickhoff, S. B. et al. A new SPM toolbox for combining probabilistic cytoarchitectonic maps and functional imaging data. Neuroimage 25, 1325-1335 (2005).

70. Worsley, K. J. et al. A unified statistical approach for determining significant signals in images of cerebral activation. Hum. Brain Mapp. 4, 58-73 (1996).

\section{Acknowledgements}

The authors are grateful to Ms. Chie Kawakami and Ms. Keiko Ueyama and the CiNet technical staff for their support during the study. The first author is also grateful to Mizuno Corporation for financial and training support for this study, especially to Mr. Kunio Yoshikawa, Ms. Naoko Matsuo, and Ms. Mikiko Kazama for their support in training, and Mr. Yasunori Kaneko and Mr. Hiroshi Nagao for their useful discussions. We would like to thank enago for English language editing.

\section{Author contributions}

Author contributions included conception and study design (E.N., T.M., H.O., C.H., and M.A.), data collection or acquisition and statistical analysis (E.N., T.M., S.H., N.K., H.O., and C.K.), interpretation of results and drafting the manuscript work or revising it critically for important intellectual content (E.N., T.M., S.H., N.K., and M.A.), and approval of final version to be published and agreement to be accountable for the integrity and accuracy of all aspects of the work (all authors).

\section{Funding}

This work was supported by JSPS KAKENHI Grant Number JP19H05723, JP19K22833 to EN, and by JSPS KAKENHI Grant Number JP20H04492 to TM. The funding sources had no involvement in the study design; in the collection, analysis, and interpretation of data; in the writing of the report; and in the decision to submit the article for publication.

\section{Competing interests}

$\mathrm{HO}$ and $\mathrm{CK}$ declare that they have financial competing interests as they are employed by Mizuno Corporation that provide a service in the form of an exercise class for older people (LaLaLa Circuit Lite). All authors declare that they have no personal association or competing interests.

\section{Additional information}

Supplementary Information The online version contains supplementary material available at https://doi.org/ 10.1038/s41598-021-02173-7.

Correspondence and requests for materials should be addressed to E.N.

Reprints and permissions information is available at www.nature.com/reprints.

Publisher's note Springer Nature remains neutral with regard to jurisdictional claims in published maps and institutional affiliations. 
(c) (i) Open Access This article is licensed under a Creative Commons Attribution 4.0 International cc) License, which permits use, sharing, adaptation, distribution and reproduction in any medium or format, as long as you give appropriate credit to the original author(s) and the source, provide a link to the Creative Commons licence, and indicate if changes were made. The images or other third party material in this article are included in the article's Creative Commons licence, unless indicated otherwise in a credit line to the material. If material is not included in the article's Creative Commons licence and your intended use is not permitted by statutory regulation or exceeds the permitted use, you will need to obtain permission directly from the copyright holder. To view a copy of this licence, visit http://creativecommons.org/licenses/by/4.0/.

(C) The Author(s) 2021 